MATEC Web of Conferences 42, 01008 (2016)

DOI: $10.1051 /$ matecconf/20164201008

(C) Owned by the authors, published by EDP Sciences, 2016

\title{
Three-Dimensional Passivity-Based Dynamic Control for Tendon-Driven Catheters
}

\author{
Minou Kouh Soltani ${ }^{1}$, Sohrab Khanmohammadi ${ }^{1}$ and Farzan Ghalichi ${ }^{2}$ \\ ${ }^{1}$ Department of Electrical and Computer Engineering at University of Tabriz, Tabriz, Iran \\ 2 Department of Biomedical Engineering at Sahand University of Technology, Tabriz, Iran
}

\begin{abstract}
This paper presents a three-dimensional dynamic model for active catheters commonly implemented in cardiac ablation, and introduces nonlinear closed-form model-based control scheme. The dynamic model includes rotational, translational and bending effects, moreover entails simple formulation to be implemented in real-time clinical application. Then, Lyapunov-based position control strategy is developed to locate the catheter tip at the desired position. Results verify the viability of the introduced approach for its applicability in robot-assisted cardiac ablation.
\end{abstract}

\section{Introduction}

Minimally invasive surgery (MIS) is a recent prevailing approach in diagnosis and treatment of percutaneous anatomical organs. Cardiac catheterization applied by steerable catheters, is one of the significant domains in MIS for electrophysiology studies, and RF ablation. Conventionally, the cardiac catheters are navigated manually by means of translating and twisting the proximal end with the bending of the distal shaft using the steering knob on the handle. Long and flexible structure of catheters along with fast cardiac motions restrain accurate positioning and consequently making stable contact with the endocardium. Hence, making stable contacts may require repetitive catheter placement that exposes both patients and surgeons into the ionizing radiation.

Currently, there are three different commercial robots that are developed to assist the catheter navigationSensei ${ }^{\circledR}$ robotic catheter system (Hansen Medical, Mountain View, CA), Niobe ${ }^{\circledR}$ (Stereotaxis, St. Louis, $\mathrm{MO}$ ), and Amigo $^{\mathrm{TM}}$ (Catheter Robotics, Inc., Mount Olive, NJ). The Sensei ${ }^{\circledR}$ allows the navigation of the catheter through the teleoperating workstation, but the platform is required to be placed close to catheterization system; therefore, it alleviates the exposure to ionizing radiation partially $[1,2]$. The Niobe ${ }^{\circledR}$ is controlled remotely with two permanent magnets located on both sides of the patient's bed. This system relies on magnetic navigation; hence, it interferes with the metallic devices implanted in the body, which restricts its employment to metallic-free environments $[3,4]$. The Amigo ${ }^{\mathrm{TM}}$ incorporates relatively less complicated and inexpensive remote catheter system, which can accommodate a range of commercially existing catheters [5]. However, all commercially available robotic systems are controlled via teleoperation devices and directed manually and failures in tracking the heart may occur due to remote manual catheter control.

To facilitate the catheterization and to realize automatic navigation, accurate position control is required. Hence, the development of dynamic spatial model of tendon-driven catheters is essential to be applicable in dynamic cardiac environment with fast motions. It should be noted that much prior work has focused in the general context of continuum robots. In the earliest studies [6, 7], Mochiyama et al. presented the general framework of kinematic and dynamic modeling of hyper-redundant flexible manipulators, while the elastic potential energy due to bending is not included in the model. Ivanescu et al. [8-10] introduced dynamic model for hyper-redundant manipulators with the assumption of two degrees of freedom in each section. In the developed model, the authors have considered the elastic potential energy in deriving the corresponding equations. In [11, 12] a Lagrangian-based 2-D dynamic model for continuum robots is established, taking into account elastic and gravity potential energies. Trivedi et al. [13] developed non-closed-form exact dynamic model for soft robotic manipulators using Cosserat rod method, while considering both end point and distributed forces on the continuum robot.

Kinematic models of continuum robots are presented based on beam theory $[14,15]$, constant curvature [16] or piecewise constant curvature modeling [17], and Cosserat rod theory $[18,19]$. Constant curvature modeling is an accurate approach provided that external loads are negligible. Cosserat rod model is the most exact kinematic model; however, the end effector pose due to the nonlinear differential equations with initial boundary 
values should be solved numerically and no closed form exact solution exist for the model.

Having developed the catheter model, the position controller should be designed for automatic catheter navigation. When the inertia effects due to small mass or low speed are negligible, kinematic controllers can be applied due to their less complication. With this assumption, in [20,21] kinematic motion controllers based on constant curvature and beam theory were presented. Dehghani et al. proposed kinematic control based on Cosserat model that has higher accuracy in the presence of external loads [22]. On the other hand, Ivanescu [9] developed fuzzy-PD controller for the simplified dynamic model with two control variables that are restricted to simulations. The recent work [23] has developed sliding mode controller for planar continuum robots based on the model presented in $[11,12]$. However, the controller suffers from chattering.

To perform model-based robot-assisted catheter navigation, a three-dimensional catheter model as well as accurate position control incorporating all designated DOFs on the catheter is required. To this end, we elaborated dynamic model of the surgical tool for tip position control, while constant curvature approach is incorporated for kinematic modeling of it, which is shown to be accurate in the presence of small payloads [24]. Based on the model, catheter tip position control is developed to precisely track a moving tissue. The rest of the paper is organized as follows. Section 2 presents the kinematics and dynamic model of the catheter. In section 3 , the spatial nonlinear Lyapunov-based position control is proposed. Control performance is simulated in section 4 , and finally the paper is concluded in section 5 .

\section{Modeling of Tendon-Driven Catheter}

\subsection{Kinematic model}

The catheter model is elaborated based on the approach originally presented by Webster and Jones [16]. It is assumed that the catheter bends with constant curvature in a single plane with no torsion about the centerline of it, which is justified through experiments [24]. In the following, with the kinematic model being described, the dynamic model will be derived.

\subsubsection{Kinematics of the distal shaft}

Fig. 1 shows a kinematic representation of tendon- driven catheters modeled with constant curvature. The elastic rod is with unstretched length $l$, and is characterized by the reference coordinate frame at the base of the distal shaft. To describe the kinematics model of the catheter, the position of each point on the distal shaft is calculated through intermediate configuration space determination, which is defined by arc parameters $(\varphi, k, l) . \varphi$ is the rotation angle from $x-z$ plane, $k$ demonstrates the curvature, and $l$ is the length of distal shaft.
Even though steerable catheters might vary in terms of external distal shaft bending mechanism, the interior framework has a common structure based on the pulling-

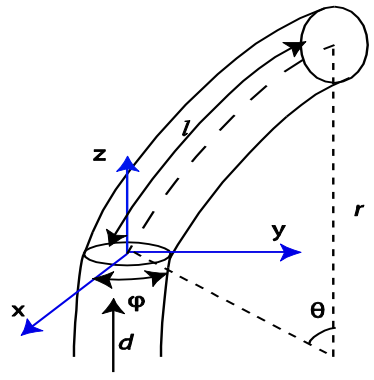

Figure 1. Geometric representation of the spatial bending of the steerable catheter, $\varphi, l, k=1 / r$ denote the out of plane rotation, length of the bending section, and the curvature, respectively.

pushing of the tendons attached to the central thin plate as depicted in Fig. 2. Typically, tendon-driven catheters are steered through three Degrees of Freedom (DOFs), handle translation $(d)$ and rotation $(\varphi)$, and distal shaft deflection $(k)$, as illustrated in Fig. 1, which is a function of rotation of the knob on the catheter handle. The transferring of forces and torques from the handle to the distal shaft is performed based on the method presented in [24]. In this work, a model of ablation catheter (SteeroCath-T, BostonScientific, Natick, MA) is adopted for the purpose of demonstration and control.

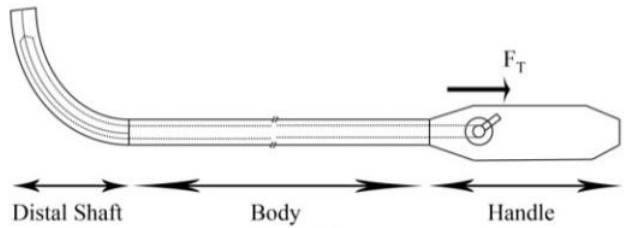

(a)

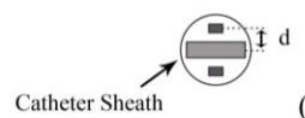

(b)

Figure 2. The schematic representation of the catheter. (a) Tendons are drawn by dashed line and the central plate (thin plate tendons attached to it) is depicted with solid line inside the distal shaft. (b) Cross section of distal shaft.

\subsection{Dynamic Model}

Dynamic model is derived using Euler-Lagrange equations

$$
\frac{d}{d t} \frac{\partial L}{\partial \dot{q}_{k}}-\frac{\partial L}{\partial q_{k}}=\tau_{k}, \quad k=1,2,3
$$

where $q_{k}$ and $\tau_{k}$ are generalized coordinates and forces, respectively, associated with each DOF on the catheter. $L$ is the Lagrangian of the system, and is defined by the difference of the kinetic and potential energy. The kinetic energy $(T)$ is the sum of translational and rotational energies defined as,

$$
\begin{aligned}
T & =T_{\text {body }}+T_{\text {distal }} \\
& =\frac{1}{2} m_{b} \dot{d}^{2}+\frac{1}{2} m_{d}\left(\dot{r}_{G}^{2}\right)+\frac{1}{2} I_{G} \dot{\theta}^{2}+\frac{1}{2} I_{r} \dot{\varphi}^{2},
\end{aligned}
$$


in which $m_{d}$ and $m_{b}$ are the mass of distal shaft and body of the catheter, respectively, $\dot{r}_{G}$ is the linear velocity of the center of mass of distal shaft, and $I_{G}$ and $I_{r}$ are the moments of inertia of the distal shaft about the bending and rotational axes, respectively. The potential energy $(U)$ is resulting from bending, gravity, and extension in continuum robots. However, since the catheter is confined to vascular on its path to the heart, the gravity force is negligible, and moreover, considering catheterization, there is no extension in the catheter, nevertheless these can be easily added to the model. Therefore

$$
U=\frac{1}{2} E I l k^{2},
$$

where $E$ is the module of elasticity, and $I$ is the second moment of area.

Substituting equation (2) and (3) into equation (1) with some manipulations leads to

$$
M(k, \varphi)\left[\begin{array}{c}
\ddot{k} \\
\ddot{\varphi} \\
\ddot{d}
\end{array}\right]+C(k, \dot{k}, \varphi, \dot{\varphi})\left[\begin{array}{c}
\dot{k} \\
\dot{\varphi} \\
\dot{d}
\end{array}\right]+G(k)=\left[\begin{array}{c}
\tau_{k} \\
\tau_{\varphi} \\
F_{d}
\end{array}\right],
$$

where $M(k, \varphi), C(k, \dot{k}, \varphi, \dot{\varphi}) \in \mathbb{R}^{3 \times 3}$ are inertia and Coriolis matrices, respectively. $\tau_{k}, \tau_{\varphi}$ and $F_{x}$ are the control inputs exerted to the corresponding DOF. The elaborated spatial dynamic model has bounded inertia matrix and skew-symmetric matrix of $\dot{M}-2 C$,

$$
\forall \xi \in \mathbb{R}^{n}, \quad \xi^{T}(\dot{M}-2 C) \xi=0,
$$

which is serving as the fundamental property for controller design.

\section{Position Control}

The objective of the robotic system is to accurately track the tip of the catheter. To this end, a 3-D nonlinear closed form passivity-based position controller is developed, where the controller is proved to be stable. The control law

$$
u=M(k, \varphi) \ddot{\xi}+C(k, \dot{k}, \varphi, \dot{\varphi}) \dot{\xi}+G(k)-k_{D} \sigma,
$$

where

$$
\left\{\begin{array}{c}
\dot{\xi}=\dot{q}_{d}-\Lambda \tilde{q}, \\
\sigma=\dot{q}-\dot{\xi}=\dot{\tilde{q}}+\Lambda \tilde{q}
\end{array}\right.
$$

and $k_{D}$ and $\Lambda$ are symmetric and positive definite matrices, is the passivity-based joint space control law. Replacing equation (6) into equation (4), the closed loop equation of the system is,

$$
M(k, \varphi) \dot{\sigma}+C(k, \dot{k}, \varphi, \dot{\varphi}) \sigma+k_{D} \sigma=0 .
$$

The Lyapunov function $V=\frac{1}{2} \sigma^{T} M(k, \varphi) \sigma+$ $\tilde{q}^{T} \Lambda k_{D} \tilde{q}$, with the time derivative $\dot{V}=\sigma^{T} M \dot{\sigma}+\frac{1}{2} \sigma^{T} \dot{M} \sigma+2 \tilde{q}^{T} \Lambda k_{D} \dot{\tilde{q}}$, and skew-symmetry property yields $\dot{V}=-\tilde{q}^{T} \Lambda^{T} k_{D} \Lambda \tilde{q}-\dot{\tilde{q}}^{T} k_{D} \dot{\tilde{q}} \leq 0$. Therefore $\tilde{q}=e=0$, is globally asymptotically stable resulting in tracking the desired joint variables accurately.
Since the catheter tip position control is the main purpose, task space control in lieu of joint space control is privileged. To adapt the passivity based approach to task space control, a new variable is defined as follows,

$$
\dot{\xi}_{p}=\dot{p}_{d}+\Lambda_{p}\left(p_{d}-p\right)
$$

where $\Lambda_{p}$ is symmetric and positive definite matrix, and

$$
\dot{\xi}=J_{v}^{-1} \dot{\xi}_{p} .
$$

$J_{v}$ is the linear velocity Jacobian. Referring back to Fig. 1 , by using the kinematic representation, the Jacobian matrix is calculated. Denoting the forward kinematics with $F$, then the Jacobian is,

$$
J_{v}=\frac{\partial F}{\partial q},
$$

Given that both generalized coordinates and end-effector position are with the same dimension $\left(q, p \in \mathbb{R}^{3}\right)$, the resulting distal section Jacobian is a square matrix.

Given that both generalized coordinates and endeffector position are with the same dimension $\left(q, p \in \mathbb{R}^{3}\right)$, the resulting distal section Jacobian is a square matrix.

Therefore, the control law in task space is defined as,

$$
u=M(k, \varphi) \ddot{\xi}+C(k, \dot{k}, \varphi, \dot{\varphi}) \dot{\xi}+G(k)+J_{v}^{T} k_{D} J_{v}(\dot{\xi}-\dot{q}),
$$

by taking derivative of equation (10), $\ddot{\xi}$ is obtained as follows,

$$
\ddot{\xi}=J_{v}^{-1} \ddot{\xi}_{p}+\dot{J}_{v}^{-1} \dot{\xi}_{p}
$$

\section{Simulations}

In order to evaluate the performance of the proposed approach, the controller is applied to a simulation of SteeroCath-T cardiac ablation catheter. The Human heart ordinarily beats $60-100$ times per minute $[25,26]$. Therefore, the designated controller should give a reasonable response within approximately $1 \mathrm{~Hz}$ for applying in such a dynamic environment.

In the simulations, the robot is modeled with the dynamic equations presented in section 2. The results of the task space position control along with the controlled joint space variables, and applied control inputs are illustrated in Figs. 3-5. The results show that the catheter tracks the moving target with high accuracy while the controlled joint space variables change in the admissible range of $k \in(0,60) \mathrm{m}^{-1}, \varphi \in(-\pi, \pi) \mathrm{m}^{-1}$. The translation limit depends on the patients' anatomical structures and the purpose of catheterization, but typically for cardiac ablation it can vary within the range of $(-50 \sim 50) \mathrm{cm}$ from the insertion point [24]. 

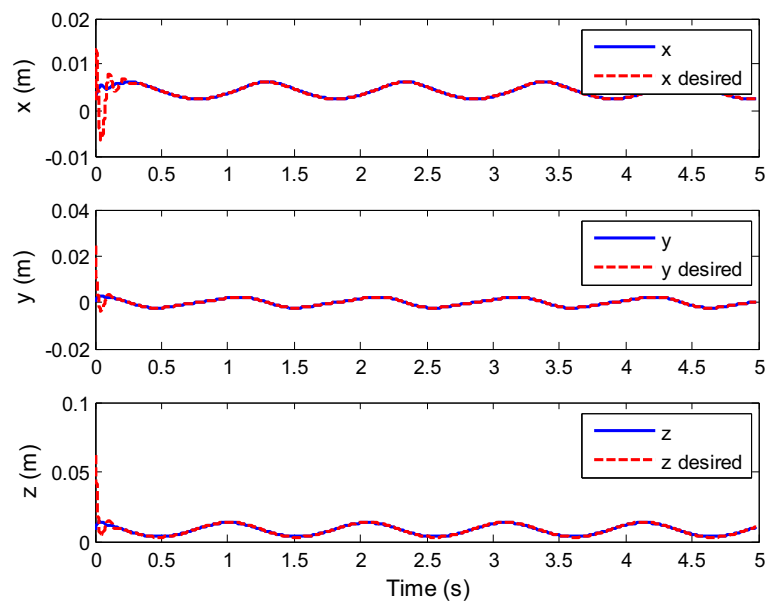

Figure 3. Passivity-based controlled distal shaft tip positionalong with desired end-effector trajectories.
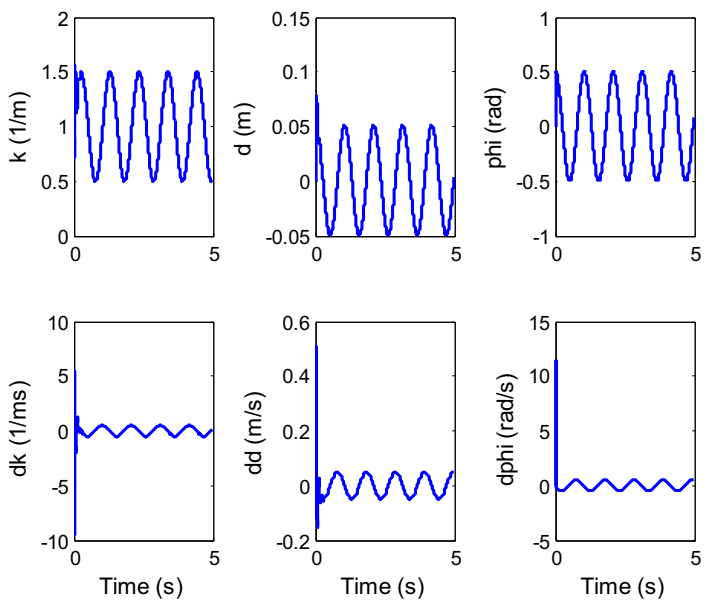

Figure 4. Passivity-based controlled joint space variables.
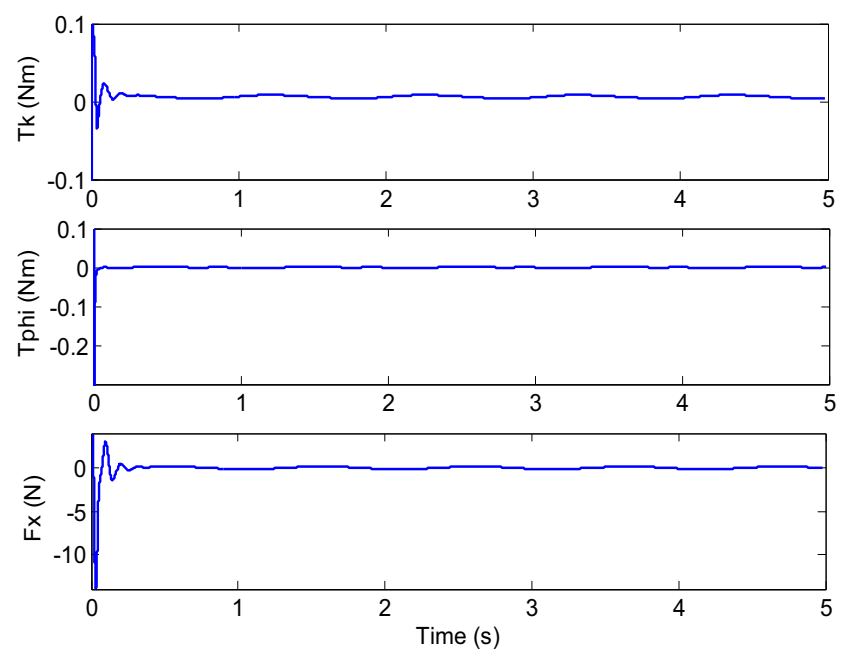

Figure 5. Outputs of the passivity-based controller.

\section{Conclusion}

This paper introduces spatial dynamic model for steerable catheters with the purpose of designing closed-form nonlinear robust controller to be applied in robot-assisted minimally invasive surgery. To the best of authors' knowledge, this is the first study applying dynamic model for the position control of tendon driven catheters providing the stability. Passivity-based control leads to enhanced robustness in contrast to inverse dynamics and feedback-linearization which is based on cancellation of nonlinearities. The controller is proved to be stable, while the performance of the controller is evaluated.

For future work, we plan to develop hybrid force/position control, and devise to elaborate multicurvature dynamic model for the catheter. Finally, the approaches would be implemented in autonomous robotassisted catheter ablation.

\section{References}

1. B.L. Nguyen, J.L. Merino, and E.S. Gang: Remote Navigation for Ablation Procedures -A New Step Forward in the Treatment of Cardiac Arrhythmias (2010).

2. W. Saliba, V.Y. Reddy, O. Wazni, J.E. Cummings, J.D. Burkhardt, M. Haissaguerre, J. Kautzner, P. Peichl, P. Neuzil, and V. Schibgilla: Atrial fibrillation ablation using a robotic catheter remote control system: initial human experience and longterm follow-up results. Journal of the American College of Cardiology, 51, 25 (2008), pp. 2407-2411.

3. Y. Fu, H. Liu, W. Huang, S. Wang, and Z. Liang: Steerable catheters in minimally invasive vascular surgery. The International Journal of Medical Robotics and Computer Assisted Surgery, 5, 4 (2009), pp. 381-391.

4. M.P. Armacost, J. Adair, T. Munger, R.R.Viswanathan, F.M. Creighton, D.T. Curd, and R. Sehra: Accurate and reproducible target navigation with the stereotaxis Niobe ${ }^{\circledR}$ magnetic navigation system. Journal of Cardiovascular Electrophysiology, 18 (s1) ( 2007), pp. S26-S31.

5. E.M. Khan, W. Frumkin, G.A. Ng, S. Neelagaru, F.M. Abi-Samra, J. Lee, M. Giudici, D. Gohn, R.A. Winkle, and J. Sussman: First experience with a novel robotic remote catheter system: Amigo ${ }^{\mathrm{TM}}$ mapping trial. Journal of Interventional Cardiac Electrophysiology, 37, 2 (2013), pp. 121-129.

6. H. Mochiyama: Hyper-flexible robotic manipulators. IEEE International Symposium on MicroNanoMechatronics and Human Science (2005), pp.41-46.M.

7. H. Mochiyama, and T. Suzuki: Kinematics and dynamics of a cable-like hyper-flexible manipulator. In proceedings of IEEE International Conference on Robotics and Automation (ICRA), 3 (2003), pp. 3672-3677.

8. M. Ivanescu, and V. Stoian: A variable structure controller for a tentacle manipulator. In Proceedings of IEEE International Conference on Robotics and Automation, 3 (1995), pp. 3155-3160.

9. M. Ivanescu: Position dynamic control for a tentacle manipulator. In proceedings of IEEE International 
Conference on Robotics and Automation (ICRA), 2 (2002), pp. 1531-1538.

10. M. Ivanescu, N. Popescu, and D. Popescu: A variable length tentacle manipulator control system. In Proceedings of the IEEE International Conference on Robotics and Automation (ICRA), 2 (2005), pp. 1531-1538.

11. E. Tatlicioglu, I.D. Walker, and D.M. Dawson: Dynamic modelling for planar extensible continuum robot manipulators. In Proceedings of the IEEE International Conference on Robotics and Automation (2007), pp. 1357-1362.

12. E. Tatlicioglu, I.D. Walker, and D.M. Dawson: New dynamic models for planar extensible continuum robot manipulators. In Proceedings of the IEEE/RSJ International Conference on Intelligent Robots and Systems (IROS) (2007), pp. 1485-1490.

13. D. Trivedi, A. Lotfi, and C.D. Rahn: Geometrically exact dynamic models for soft robotic manipulators. In Proceedings of the IEEE/RSJ International Conference on Intelligent Robots and Systems (IROS) (2007), pp. 1497-1502.

14. D.B. Camarillo, C.F. Milne, C.R. Carlson, M.R. Zinn, and J.K. Salisbury: Mechanics modeling of tendondriven continuum manipulators. Robotics, IEEE Transactions on, 24, 6 (2008), pp. 1262-1273.

15. M. Khoshnam, M. Azizian, and R.V. Patel: Modeling of a steerable catheter based on beam theory. 2012 IEEE International Conference on Robotics and Automation (ICRA) (2012), pp. 46814686.

16. R.J. Webster, and B.A. Jones: Design and kinematic modeling of constant curvature continuum robots: A review. The International Journal of Robotics Research, 29 (2010), pp. 1661 -1683.

17. T. Mahl, A.E. Mayer, A. Hildebrandt, and O. Sawodny: A variable curvature modeling approach for kinematic control of continuum manipulators. In Proceedings of the American Control Conference (ACC), Washington, DC (2013), pp. 4945-4950.

18. B.A. Jones, R.L. Gray, and K. Turlapati: Three dimensional statics for continuum robotic. In Proceedings of the IEEE/RSJ International Conference on Intelligent Robots and Systems (IROS), St. Louis, MO (2009), pp. 2659-2664.

19. D. Trivedi, A. Lotfi, and, C.D. Rahn: Geometrically exact models for soft robotic manipulators. IEEE Transactions on Robotics, 24, 4 (2008), pp. 773-780.

20. A. Kapoor, N. Simaan, and R.H. Taylor: Suturing in confined spaces: constrained motion control of a hybrid 8-DoF robot. In Proceedings of International Conference on Advanced Robotics (2005), pp. 452459.

21. D.B. Camarillo, C.R. Carlson, and J.K. Salisbury: Configuration tracking for continuum manipulators with coupled tendon drive. IEEE Transactions on Robotics, 25, 4 (2009), pp. 798-808.

22. M. Dehghani, and S. A. A. Moosavian: Modeling and control of a planar continuum robot. In Proceedings of IEEE/ASME International Conference on Advanced Intelligent Mechatronics (AIM) (2011), pp. 66-971.
23. A.D. Kapadia, I.D. Walker, D.M. Dawson: A modelbased sliding mode controller for extensible continuum robots. In Proceedings of the 9th WSEAS international conference on Signal processing, robotics and automation. World Scientific and Engineering Academy and Society (WSEAS) (2010), pp. 113-120.

24. Y. Ganji, and F. Janabi-Sharifi: Kinematic characterization of a cardiac ablation catheter. In Proceedings of IEEE/RSJ International Conference on Intelligent Robots and Systems (IROS) (2007), pp. 1876-1881.

25. S.B. Kesner, and R. D. Howe: Force control of flexible catheter robots for beating heart surgery. In Proceedings of IEEE International Conference on Robotics and Automation (ICRA) (2011), pp. 15891594.

26. M. H. Beers, A. Fletcher, T. Jones, R. Porter, M. Berkwitz, and J. Kaplan, The Merck Manual of Med. Inf., Merck Research Laboratories, (2003). 